\title{
Threshold Policies for Single-Resource Reservation Systems
}

\author{
E. G. Coffman Jr. Predrag Jelenkovic \\ Columbia University, New York
}

\section{Introduction}

Requests for a resource arrive at rate $\lambda$, each request specifying a future time interval, called a reservation interval, to be booked for its use of the resource. The advance notices (delays before reservation intervals are to begin) are independent and drawn from a distribution $A(z)$. The durations of reservation intervals are sampled from the distribution $B(z)$ and are independent of each other and the advance notices. We let $\mathrm{A}$ and $\mathrm{B}$ denote random variables with the distributions $A(z)$ and $B(z)$ (the functional notation will always allow one to distinguish between our two uses of the symbols $A$ and $B$ ).

The following greedy reservation policy was analyzed in [3]: A request is immediately accepted (booked) if and only if the resource will be available throughout its reservation interval, i.e., the resource has not already been reserved for a time period overlapping the requested reservation interval. In [3], the authors compute an efficiency measure, called the reservation probability, which is the fraction of time the resource is in use.

This paper studies the reservation probability for a more general greedy policy of threshold type that is defined by two parameters $s$ and $\tau$. If a request has an advance notice less than $s$ or a duration exceeding $\tau$, then the threshold policy makes an attempt to book it under the greedy rule; otherwise, it is rejected even if it could have been accommodated. Our main result is an expression for the asymptotic reservation probability as $s \rightarrow \infty$ and the advance-notice distribution becomes progressively more spread out.

The above result relates asymptotics of reservation policies to asymptotics of interval packing policies, a connection first studied in [3]. In the interval packing problem [1], intervals arrive randomly in $\mathbf{R}_{+}^{2}$ according to a Poisson process in the two dimensions representing arrival times $t$ and the left endpoints of the arriving intervals. Interval lengths are i.i.d., and since we will map them to reservation intervals, we let their distribution also be denoted by $B(z)$. The intensity is 1 , i.e., an average of one interval arrives per unit time per unit distance. For a given $x>0$, an arriving interval is packed (or accepted) in the 'containing' interval $[0, x]$ under the greedy algorithm if and only if it is a subinterval of $[0, x]$ and it does not overlap an interval already accepted. The problem is to find, or at least estimate, the function $K(t, x)$, which is the expected total length of the intervals accepted by the greedy policy during $[0, t]$, assuming that none has yet been accepted by time $0([0, x]$ is initially empty).
Estimates of $K(t, x)$ were obtained in [3] from its Laplace transform $\mathcal{K}(t, u)$; these results are special cases of the corresponding results for the threshold packing policy with parameters $s, \tau$. The threshold packing policy extends greedy interval packing much as we extended the greedy reservation policy: An interval is processed by the greedy packing algorithm if its length is at least $\tau$ or if it arrives no sooner than $s$; otherwise, it is rejected. The next section exhibits the Laplace transform of $H_{\tau}(s, t, x)$, the expected total length of the intervals accepted during $[0, t], t \geq s$, by the threshold packing policy with parameters $s, r$. Note that threshold packing reduces to simple greedy packing if $\tau=0$ or if $s=0$. The formulas in the next section will verify that $K(t, x)=H_{0}(t, t, x)$.

As noted in [3], there are many potential applications covered by models like ours. However, relatively new applications in existing and proposed communication systems, e.g., teleconferencing and video-on-demand systems, have given a fresh impetus to research on reservation systems. Previous work in the communications field is quite recent and focuses more on engineering problems than mathematical foundations; past research has dealt with the implementation issues of incorporating distributed advance-notice reservation protocols in current networks, and with the algorithmic issues concerned with well utilized resources in reservation systems (see $[3,4,5]$ for many references). For the analysis of mathematical models different from our own, see the work of Virtamo [5] and Greenberg, Srikant, and Whitt [4].

\section{Threshold interval packing}

Let $b_{\tau}:=\mathbf{E} B \cdot 1(B>\tau), b_{\tau}^{(2)}:=\mathbf{E} B^{2} \cdot 1(B>\tau)$, and $p=$ $p_{\tau}:=\mathbf{P}(B>\tau)$. Denote by $L_{\tau}(s, t, x)$ the total length of the intervals packed at time $t \geq s$ in $[0, x]$, and let $H_{\tau}(s, t, x):=$ $\mathbf{E} L_{\tau}(s, t, x)$.

We compute the rate of change of $H_{\tau}(s, t, x)$ with respect to $s$ for $x \geq d$ by expressing $H_{\tau}(s+\Delta s, t, x)$ in terms of $H_{\tau}(s, t, x)$ and the events occurring in the time interval $[0, \Delta s]$. On doing so, rearranging the recurrence and taking the limit $\Delta \rightarrow 0$, we obtain the integro-differential equation

$$
\begin{aligned}
\frac{\partial H_{\tau}(s, t, x)}{\partial s}= & -\left(p x-b_{\tau}\right) H_{\tau}(s, t, x)+b_{\tau} x-b_{\tau}^{(2)} \\
& +2 \int_{\tau}^{d} d B(z) \int_{0}^{x-z} H_{\tau}(s, t, y) d y .(1)
\end{aligned}
$$


Note the boundary condition $K(t, x)=H_{0}(t, t, x)$, the known result for the simple greedy rule. Note also that the effect on (1) of putting $\tau=0$ is confined to constants depending only on $\tau$. Thus, exactly the same analysis for $K$ in [3] can be applied to $H_{\tau}$ here. We introduce the transforms $\mathcal{H}_{\tau}(s, t, u):=\int_{d}^{\infty} e^{-u x} H_{\tau}(s, t, x) d x, \mathcal{B}_{\tau}(u):=$ $\int_{\tau}^{d} e^{-u z} d B(z)$, and then transform (1) to obtain a pde whose solution is readily found to be

$$
\begin{array}{r}
\mathcal{H}_{\tau}(s, t, u)=\frac{1}{u^{2}} \int_{0}^{s} \mathcal{C}_{\tau}(s-z, t, u+p z) \mathcal{G}_{\tau}(u, p z) d z(2) \\
+\left(\frac{u+p s}{u}\right)^{2} \mathcal{K}(t-s, u+p s) \mathcal{G}_{\tau}(u, p s), x \geq d
\end{array}
$$

where $\mathcal{G}_{\tau}(u, v):=\exp \left(b_{\tau} v-2 \int_{u}^{u+v} \frac{1-\mathcal{B}_{\tau}(y)}{y} d y\right)$, and where $\mathcal{C}_{\tau}(s, t, u)$ is the transform of a well-behaved function easily computed by a recursive procedure. (See the expanded version of the paper [2] for details.) As illustrated below, explicit expressions are available for simple distributions $B($.$) .$

Estimates of $H_{\tau}$. Leading-term asymptotics in $x$ are given by the following result, in which

$$
\begin{aligned}
\alpha_{\tau}(s, t):= & \int_{0}^{s} \mathcal{C}_{\tau}(s-z, t, p z) \mathcal{G}_{\tau}(p z) d z \\
& +\mathcal{K}(t, p s)(p s)^{2} \mathcal{G}_{\tau}(p s)
\end{aligned}
$$

Theorem 1 For any $s>0, t \geq 0, H(s, t, x) \sim$ $\alpha_{\tau}(s, t) x$ as $x \rightarrow \infty$.

Proof sketch: One first verifies that the functions $\mathcal{C}_{\tau}, \mathcal{G}_{\tau}$, and $\mathcal{K}$ are such that $\mathcal{H}_{\tau}$ in (2) satisfies $\mathcal{H}_{\tau}(s, t, u) \sim \alpha_{\tau}(s, t) / u^{2}$ as $u \downarrow 0$.. An application of Karamata's Tauberian theorem and routine manipulations then proves the theorem.

Example 1: Consider the case where interval lengths have only the values 1 or 2 , with probabilities $\mathbf{P}(B=2)=1-$ $\mathbf{P}(B=1)=q$. Computations give

$$
\mathcal{G}(u, v)=\exp \left(2 q-2 \int_{u}^{u+v} \frac{1-q e^{-2 y}}{y} d y\right)
$$

and

$\mathcal{C}_{\tau}=2 q e^{-2 u}\left(1+u\left[\frac{e^{-u}-e^{-2 u}}{u}-\frac{e^{-u}-e^{-2 u-(1-p) t}}{u+(1-p) t}\right]\right)$,

from which we obtain $\mathcal{K}(t, u)$ and hence an explicit integral formula for $\alpha(s, t)$ in (3) that we can evaluate numerically.

For $t=7, p=0.4$, we used Mathematica to compute the curve for $\alpha_{\tau}(s, 10-s)$. The threshold algorithm is strictly better than the greedy policy $\left(\alpha_{\tau}(0,10)\right)$ for a large range of values of the threshold parameter $s$.

In order to obtain higher-order error terms, we use complex analysis and the Cauchy residue theorem to evaluate directly the Laplace-transform inversion formula for $\mathcal{H}_{\tau}$. This gives the main result needed for the limit law of the next section,

Theorem 2 For any fixed $\xi, T>0$ with $\xi>2 T>0$, there exists a constant $\gamma_{\tau}(t)$, such that

$$
\sup _{0 \leq t_{1}, t_{2} \leq T}\left|H_{\tau}\left(t_{1}, t_{2}, x\right)-\left(\alpha_{\tau}(t) x+\gamma_{\tau}(t)\right)\right|=O\left(e^{-\xi x}\right) .
$$

\section{Advanced-notice limit law}

Let the advance notice distribution be uniform on $[0, a]$ and for a given $\delta,(0<\delta<1)$ consider the threshold reservation policy: If the advance notice of a request is in $[\delta a, a]$ and the duration requested is less than a given $\tau$, then the request is rejected. Otherwise, it is processed according to the greedy reservation policy (i.e., it is accepted if does not overlap a time interval already reserved). Let $P_{a}(\lambda, \tau, \delta)$ denote the reservation probability, i.e., the stationary probability that the resource is in use.

Theorem 3 As the support of the advance notice distribution tends to infinity $(a \rightarrow \infty)$, we have the asymptotic reservation probability $P_{a}(\lambda, \tau, \delta) \sim \alpha_{\tau}(\delta \lambda, \lambda)$.

The proof follows the approach used in [3] to establish the analogous result for the simple greedy policy.

\section{References}

[1] E. G. Coffman, Jr., Leopold Flatto, Predrag Jelenkovic, and Bjorn Poonen. Packing random intervals on line. Algorithmica, 22(1998), 448-476.

[2] E. G. Coffman, Jr. and Predrag Jelenkovic. Threshold policies for interval packing and reservation systems. Technical Report, Electrical Engineering Dept., Columbia University, New York, NY 10027.

[3] E. G. Coffman, Jr., Predrag Jelenkovic, and Bjorn Poonen. Reservation Probabilities. Adv. Perf. Anal., 2(1999), 129-158.

[4] A. Greenberg, R. Srikant, and W. Whitt. Resource sharing for book-ahead and instantaneous- request calls. Proceedings, ITC 15, pages 539-548, Washington, D.C., June 1997.

[5] J. T. Virtamo. A model of reservation systems. IEEE Trans. Comm. Sys., 40(1992), 109-118. 